\title{
Patterns of intestinal schistosomiasis among mothers and young children from Lake Albert, Uganda: water contact and social networks inferred from wearable global positioning system dataloggers
}

\author{
Edmund Y. W. Seto ${ }^{1}$, José C. Sousa-Figueiredo ${ }^{2,3}$, Martha Betson $^{2}$, Chris Byalero, ${ }^{4, *}$, Narcis B. \\ Kabatereine $^{4}$, J. Russell Stothard ${ }^{2}$ \\ ${ }^{1}$ School of Public Health, University of California, Berkeley, CA 94720, United States of America; ${ }^{2}$ Disease \\ Control Strategy Group, Liverpool School of Tropical Medicine, Pembroke Place, Liverpool, L3 5QA, United \\ Kingdom; ${ }^{3}$ Department of Infectious and Tropical Diseases, London School of Hygiene and Tropical Medicine, \\ Keppel Street, London, WC1E 7HT, United Kingdom; ${ }^{4}$ Vector Control Division, Ministry of Health, P.O. Box \\ 1661, Kampala, Uganda; "Deceased May 2012
}

\begin{abstract}
The establishment of a national control programme (NCP) in Uganda has led to routine treatment of intestinal schistosomiasis with praziquantel in the communities along Lake Albert. However, because regular water contact remains a way of life for these populations, re-infection continues to mitigate the sustainability of the chemotherapy-based programme. A six-month longitudinal study was conducted in one Lake Albert community with the aim of characterizing water contact exposure and infection among mothers and their young preschool-aged children as the latter are not yet formally included within the NCP. At baseline the cohort of 37 mothers, 36 preschool-aged children had infection prevalences of $62 \%$ and $67 \%$, respectively, which diminished to $20 \%$ and $29 \%$, respectively, at the 6 -month post-treatment follow-up. The subjects wore global positioning system (GPS) datalogging devices over a 3-day period shortly after baseline, allowing for the estimation of time spent at the lakeshore as an exposure metric, which was found to be associated with prevalence at follow-up $(\mathrm{OR}=2.1$, $\mathrm{P}=0.01$ for both mothers and young children and odds ratio $(\mathrm{OR})=4.4, \mathrm{P}=0.01$ for young children alone). A social network of interpersonal interactions was also derived from the GPS data, and the exposures were positively associated both with the number and duration of peer interaction, suggesting the importance of socio-cultural factors associated with water contact behaviour. The findings illustrate reduction in both prevalence and intensity of infection in this community after treatment as well as remarkably high rates of water contact exposure and re-infection, particularly among younger children. We believe that this should now be formally considered within NCP, which may benefit from more in-depth ethnographic exploration of factors related to water contact as this should provide new opportunities for sustaining control.
\end{abstract}

Keywords: Schistosoma mansoni, household contact, water contact behaviours, exposure, spatial-temporal interactions, global positioning system, Uganda.

Introduction

Schistosomiasis is a snail-transmitted, water-borne parasitic disease infecting an estimated 207 million people worldwide (Steinmann et al., 2006). Over 95\% of the present global distribution is concentrated in sub-Saharan Africa (SSA) where the disease is ranked second only to malaria in public health importance (Chitsulo et al., 2000). In SSA there are broadly two forms of schistosomiasis, intestinal and urogenital; the

Corresponding author:

J. Russell Stothard

Disease Control Strategy Group

Liverpool School of Tropical Medicine

Pembroke Place, Liverpool, L3 5QA, United Kingdom

Tel.:+44 1517053724

E-mail: jrstoth@liv.ac.uk latter resulting from infection with Schistosoma haematobium mainly causing haematuria (in 70 million) and urinary and genital tract pathology (in 106 million), while the former results from infection with Schistosoma mansoni and estimated to cause significant morbidity as chronic hepatomegaly (in 8.5 million), regular blood in the stool (in 4.4 million) and persistent diarrhoea (in 0.78 million) (van der Werf et al., 2003). To counter these detrimental effects, increased international support has now fostered several national control programmes (NCPs) delivering relatively inexpensive chemotherapy to afflicted individuals, as well as to at-risk communities. With the firm intention of reducing morbidity, preventive chemotherapy is strongly advocated by the World Health Organization (WHO) but as treatment does not prevent re-infection, continuation of activities 
and/or lifestyle which promote exposure to contaminated water erodes the impact of medication (Utzinger et al., 2009).

In Uganda, both intestinal and urogential schistosomiasis occur, but they have very different geographical distributions (Brooker et al., 2009). Urogenital schistosomiasis is focused in the central region of the country, whereas intestinal schistosomiasis is more widespread, being particularly intense on the shoreline environments of Lake Victoria and Lake Albert as well as along the Nile (Kabatereine et al., 1992, 1996; Balen et al., 2006; Brooker et al., 2008). As part of the country's health sector strategic plan, a NCP was put into action in 2003 with primary emphasis upon preventive chemotherapy; praziquantel (for schistosomiasis) and albendazole (for soil-transmitted helminthiasis) which is given out annually and en-masse to children of school-age, principally utilizing the Uganda's universal primary education (UPE) infrastructure (Hotez et al., 2007; Kabatereine et al., 2007).

This NCP is coordinated by the Ministry of Health's Vector Control Division, which initially received support from the Bill \& Melinda Gates Foundation and subsequently from USAID (Fleming et al., 2009). Whilst several million children have received regular treatment - a major achievement - the remaining challenge is to ensure programme sustainability and effective long-term drug coverage. However, integration of these activities with improvements in, and modifications of, environmental and behavioural factors is needed to ultimately lower parasite transmission (Utzinger et al., 2003). For example, a more enduring solution is needed for the Lake Albert shoreline as prevalence of infections remains high in primary school children $(>50 \%)$ despite annual chemotherapy. In addition, the disease is also present in much younger children with many patently infected even by their third year of life. As yet, these younger children are not formally included within the treatment remit of the NCP, which has created an unfortunate health inequity (Johansen et al., 2007; Stothard and Gabrielli, 2007a,b). To readdress this, a detailed research study commenced in November 2008 investigating the infection dynamics within the younger child before and after treatment as well as identifying causal risk-factors (Sousa-Figueiredo et al., 2010; Stothard et al., 2011a).

As the majority of the people on Lake Albert are directly associated with the local fishing industry, elevated levels of human water contact are an immediate reason why disease transmission is particularly intense there (de Moira et al., 2007). Combined with all too few potable water sources, household water is drawn directly and daily from the lake for domestic use. In addition, people use the lake for cleaning, washing and bathing. Detailed snail surveys have revealed local abundance of Biomphalaria, with both Biomphalaria stanleyi and B. sudanica being particularly permissive intermediate hosts for S. mansoni (Kazibwe et al., 2006, 2010). Similarly, as local sanitation is very poor, faecal material frequently contaminates the lake resulting in snails shedding cercariae throughout the year (Kazibwe et al., 2010). Indeed, for a long time this part of Uganda has been well-known for a particularly aggressive form of intestinal schistosomiasis, precipitating the death of many adolescent and adults (Kabatereine et al., 1992, 1996; Balen et al., 2006).

Assessing individual-level risk of schistosomiasis infection is a challenge, involving the same biases which are pervasive throughout environmental epidemiology. Accurately characterizing where, when and how human exposure to cercariae occurs during periods of water contact are critical to understanding the process. This is particularly true for exposures in younger children where the infection dynamics in this age class are just beginning to be explored. While various methods have been used to assess exposure previously, including self-reported exposure questionnaires and direct observations (Kabatereine et al., 1999; Gazzinelli et al., 2001; Scott et al., 2003; Spear et al., 2004; Seto et al., 2007a), each has its limitations and shortcomings. Self-reporting questionnaires are problematic due to inaccurate recall and deliberate misreporting as well as not being applicable to young children who cannot read and write. While direct observation studies are more informative, they can be more logistically challenging due to the requirements of several field personnel, each of whom observe often numerous study participants conducting rather intricate water contact behaviour. With both methods there are largely unavoidable concerns that behaviour may be altered by the nature of the assessment, a socalled "experimenter effect" (Barber and Silver, 1968).

A previous study of wearable global positioning system (GPS) receivers (Seto et al., 2007b) suggests a promising alternative to traditional methods of exposure investigation. Datalogging capacities, where time, position and velocity can be recorded, usher in new possibilities for real-time monitoring and spatio-temporal recording. Advances in miniaturization, waterproofing, and perhaps most importantly, reductions in unit purchase price, make this technology particularly attractive for epidemiological studies. Programmable GPS dataloggers can now be bought "off-the- 
shelf" and record complex time-activity patterns of individuals in great detail. Similar units, used in previous vector-borne disease studies, have been evaluated in terms of spatial accuracy and acceptability for epidemiologic work (Vazquez-Prokopec et al., 2009; PazSoldan et al., 2010). For schistosomiasis studies, wearable GPS dataloggers could provide objective assessments of the levels of water contact and, as several people can be monitored simultaneously, identify more complex and cryptic interactions between individuals, for example, similarities or differences between community cross-sections or presence and strength of interactions in social networks. For instance, certain social networks may be important factors leading to unhealthy behaviours (e.g. sexually transmitted diseases (Rothenberg, 2009) and smoking (Christakis and Fowler, 2008)), providing additional context to further exposure. Background on social networks and social network analysis may be found in Scott (1988). With respect to schistosomiasis, one possible example could be the social ties between mothers within a community that influence when and where lake water is drawn for household use. Such cryptic interactions are difficult to document precisely using traditional approaches, but can be attempted based upon coincidence between individuals' time-activity patterns.

Here, we present a pilot longitudinal study conducted in a schistosomiasis-endemic community in Lake Albert, Uganda, in which wearable GPS units were used to assess exposure, in particular referencing the spatial and activity patterns of mothers and their young children and their acceptance of these wearable GPS devices. We used GPS data to estimate exposures and their associations with infection with $S$. mansoni and the levels of self-reported measures of water contact. For contrast, we also present data from a smaller cohort of school-aged children and adult fishermen, to create a cross-sectional comparator for the spatial micro-epidemiology of risk along the Lake Albert shoreline.

\section{Materials and methods}

\section{Study area}

The study was conducted in Bugoigo, an impoveished fishing village on the northeastern shore of Lake Albert in Buliisa district, Uganda. The village has been the base for many research projects investigating the immunology and epidemiology of intestinal schistosomiasis. The snail ecology of this transmission area has been described in detail by Kazibwe et al. (2006) establishing that $B$. stanleyi and B. sudanica both serve as permissive intermediate hosts for $S$. mansoni. The snails occupy slightly different aquatic niches, the former preferring deeper water habitats, while the latter is more frequently found along the shallow lake shoreline margins. The population dynamics of both species undergo seasonal fluctuations.

\section{Patient cohort at baseline}

A patient cohort consisting of 53 pairs of mothers with young children (aged $<6$ years) was randomly recruited after obtaining informed consent from households participating in a broader epidemiological study investigating schistosomiasis in young children. Additionally, for local comparison, a smaller group of 15 schoolchildren (aged 6-14 years) and 10 adult fishermen were asked to participate in this GPS study. Upon selection, all participants were asked to complete a baseline survey to establish age, sex, and history of schistosomiasis infection and treatment.

To ascertain their parasitological status, each participant submitted stool samples at baseline for examination of S. mansoni infection using the Kato-Katz thick smear procedure (Katz et al., 1972). Two smears were made per received stool sample and viewed under the microscope to determine egg counts at 100x magnification. Egg counts were converted to eggs per gram (EPG) of faeces, and analysed as either positive for infection (> 0 EPG) or according to the established categories of "light" $(\mathrm{EPG}<100)$, "moderate" $(\mathrm{EPG}=$ 100-399), or "heavy" (EPG $\geq 400$ ) infection (WHO, 2002). To bolster this diagnosis by microscopy, urine samples from each participant were also tested using a rapid diagnostic test measuring the excretion of the circulating cathodic antigen (CCA) in the urine (Rapid Medical Diagnostics, Pretoria, South Africa) as an alternative. After examination, all persons were treated with standard doses of praziquantel and albendazole regardless of infection status.

\section{Wearable GPS system dataloggers}

After completing the baseline survey and examination, the participants were asked to wear a GPS datalogger (I-gotU GT-120, Mobile Action, UK; dimension $44.5 \times 28.5 \times 13 \mathrm{~mm}$, weight $20 \mathrm{~g}$ ) for three consecutive days. A custom-designed elasticised fabric strap with Velcro fastener was made to house the GPS securely. The GPS device was first placed within a sealable plastic bag to best ensure further waterproofing, before the unit and strap were worn on the arm or 


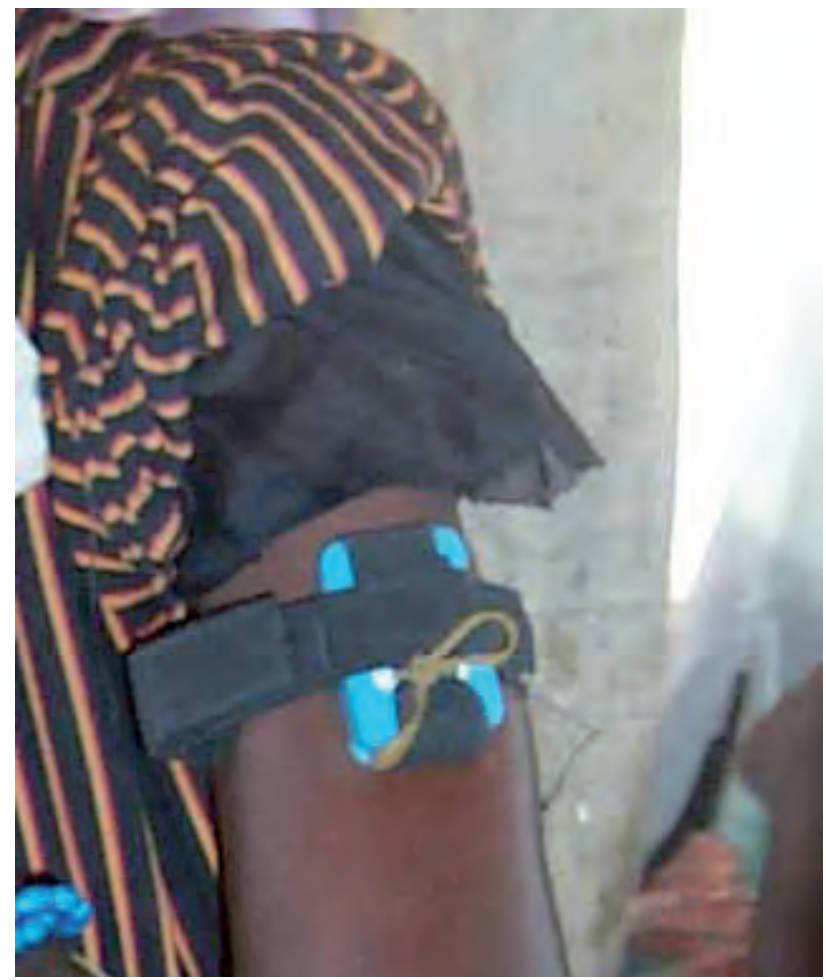

Fig. 1. GPS datalogger with custom holder worn on the left upper arm. The arm-strap has two metal D-loops with foldback Velcro fastener for custom tightening. The elasticised fabric contains the GPS device set within a cruciform support and further firmly held in place with a tied rubber band. To ensure full waterproofing the device is first placed within a sealable plastic bag before setting within its silicone rubber sleeve.

wrist (Fig. 1). Participants were instructed to wear the GPS at all waking hours, including those times spent swimming, bathing, washing clothes, cleaning, fishing, playing and/or working. The unit was powered by a rechargeable $230 \mathrm{mAh}$ Lithium-ion battery that allowing for GPS datalogging at 1-min intervals for the entirety of the 3-day period.

Owing to availability of GPS units, the study was spread out over six separate periods (four periods for pairs of mothers and their young children, one for school-aged children and one for fishermen). All participants were asked a series of questions when they returned the GPS instruments aimed at assessing user acceptance, self-reported admission of whether lake water contact occurred during the period the GPS was worn, and if so, the duration in minutes of the water contact. After each study period, the dataloggers were collected, and data downloaded to a personal computer for mapping and assessment of exposure. The processing included conversion of the native GPX files to shape-files for analysis in an ArcGIS (ESRI, Redlands, CA, USA) geographical information system (GIS).

\section{Patient cohort at 6-month follow-up}

Six months after the baseline survey, a follow-up examination was conducted on mothers and young children alone, as they are part of an ongoing cohort study (Stothard et al., 2011a). Fishermen and schoolaged children were not re-examined as most were absent upon follow-up. Kato-Katz examinations were carried out as well as urine-CCA testing, and the participants were again treated with praziquantel and albendazole regardless of infection status.

\section{Exposure assessment}

In the GIS, the data were first filtered according to the velocity filtering method suggested by Seto et al. (2007b), removing noisy data with speeds greater than or equal to $1 \mathrm{~m} / \mathrm{s}$. This velocity was chosen based on the general lack of motorised transportation, and our focus on water contact along the shoreline, rather than studies of mobility. Put simply, this approach is based on a comparison of the distance (or speed) traveled between successive GPS measurements. If the speed traveled is greater than a specified threshold filter value (in this case $1 \mathrm{~m} / \mathrm{s}$ ), we removed the measurement (Seto et al., 2007b). Further processing in GIS included the generation of kernel densities and regularly spaced contours (at intervals of 0.02 points per $\mathrm{m}^{2}$ ) to aid in the visualization of the GPS point clouds.

Satellite data from the tri-decadal orthorectified Landsat TM mosaic product (USGS, Reston, VA, USA) were used to establish the geographical location of the shoreline for our study area. The shoreline was traced from the $15 \mathrm{~m}$ resolution satellite image, and confirmed against GPS coordinates collected by motorcycle along the shoreline in the field. A $100 \mathrm{~m}$ buffer area was created on the lake side of the shoreline to represent the area in which water contact might result in infection. This was verified on the ground by wading at the lake margins; it should be noted that at the time of the survey the lake surface was particularly low with depths no more than $25 \mathrm{~cm}$ at $25-50 \mathrm{~m}$ from the shoreline. Numerous snail populations were encountered within this buffer distance (Kazibwe et al., 2010).

Exposure was quantified in two ways. First, by computing the duration of exposure in the shoreline risk area for each participant by intersecting the GPS points recorded by their datalogger with the aforementioned buffer area in GIS; second, by considering the time of the day in which the activity occurred within the risk area weighting the data based on hourly cercarial shedding data for snails in the Lake Albert region (Kazibwe et al., 
2010). Our weighting scheme was based on the proportion of total infective cercariae during the entire day that would be present at any given hour of the day, assuming that cercariae would remain maximally infective for approximately an additional hour after shedding (hence the slight time lag in the weighting compared to the shedding data in Fig. 2). The weights were normalised to sum to one. This weight was multiplied with each duration of GPS activity in the risk area to create a weighted exposure score. The sum of exposure minutes and weighted exposure scores were generated for each participant and summarised below for each cohort group. Differences in exposure between mother and child were assessed using the Wilcoxon matched-pairs signed-ranks test in Stata version 10 (StataCorp, College Station, TX, USA). Additionally, univariate regression models were used to search for relationships between infection and GPS-derived exposure. The statistical significance was defined as less than 0.05 probability.

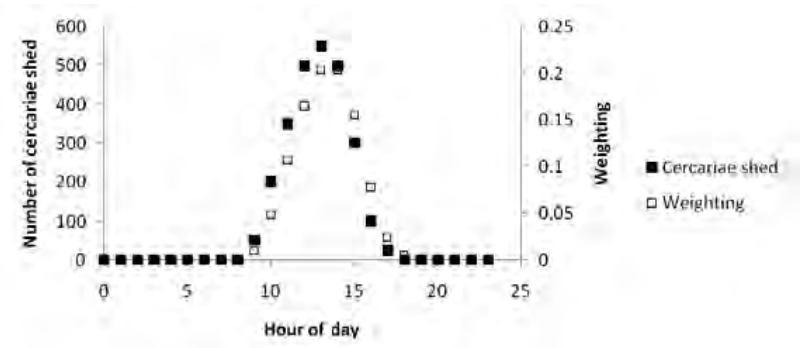

Fig. 2. Numbers of cercariae shed from snails by time of day based upon data from Kazibwe et al. (2010), and then converted into a risk weighting-factor.

\section{Social interaction}

Based on the temporal sequence of GPS data, we defined a social interaction in the exposure zone as two persons spatially near each other (within $50 \mathrm{~m}$, which places people in conversational distance after accounting for some inaccuracy in the GPS data). Social interaction was quantified in two ways. First, for each person, we computed the number of persons he/she interacted with in the exposure zone; second, we computed the total amount of time he/she interacted with others in the exposure zone. Social networks were visualised using Gephi, an open source software for network analysis (Bastian et al., 2009).

\section{Ethical approval}

This study was approved by the London School of Hygiene and Tropical Medicine and the Uganda Council for Science and Technology. Each recruited subject was provided with a study sensitization-leaflet showing the GPS device and information on how it would be worn if they chose to participate. Oral informed consent was provided before providing written informed consent. To ensure recovery of GPS devices, an incentive of 5,000 Schillings ( 2.5 US\$) was provided to compensate for the participants time. At the end of the study, each participant received the standard dose of praziquantel $(40 \mathrm{mg} / \mathrm{kg}$ bodyweight) and albendazole $(400 \mathrm{mg}$ for all over the age of two years and $200 \mathrm{mg}$ for infants under the age of two years) regardless of their infection status.

\section{Results}

\section{Baseline and 6-month follow-up}

Age, sex, and past infection and treatment profiles for the four groups are shown in Table 1. The responses to past infection and treatment reflect the high endemicity of $S$. mansoni within in this area. All fishermen and the majority of mothers and school-aged children reported that they had been previously infected and treated. School-aged children reported a greater percentage of past treatment than past infection/diagnosis, which is indicative of the school-based treatment programmes with delivery of treatment to all on the basis of diagnosis in a sentinel sub-set.

Table 1. Study participants at baseline with reported past history of diagnosis and intestinal schistosomiasis treatment.

\begin{tabular}{lcccc}
\hline Group & N & $\begin{array}{c}\text { Mean age } \\
\text { (SD; range) }\end{array}$ & \% male & $\begin{array}{c}\text { \% report } \\
\text { past S. mansoni } \\
\text { infection } \\
\text { past treatment } \\
\text { for S. mansoni }\end{array}$ \\
\hline Mothers & 37 & $31(8.1 ; 20-47)$ & 0 & 60 \\
Young children & 36 & $3.7(1.0 ; 1-5)$ & 56 & NA \\
School-aged children & 15 & $10(2.5 ; 7-14)$ & 47 & 67 \\
Fishermen & 9 & $38(11 ; 24-59)$ & 100 & 100 \\
\hline
\end{tabular}

NA, not applicate 
Table 2. Prevalence of S. mansoni infection and infection intensity at baseline and at 6-month follow-up.

\begin{tabular}{|c|c|c|c|c|c|c|}
\hline \multirow{2}{*}{ Group } & \multicolumn{6}{|c|}{ Baseline } \\
\hline & $\mathrm{N}$ & Infection $(\%)$ & Light $(\%)$ & Moderate (\%) & Heavy $(\%)$ & Mean EPG (SD, range) \\
\hline Mothers & 37 & 62 & 68 & 19 & 13 & $\begin{array}{c}225 \\
(533 ; 0-2,550)\end{array}$ \\
\hline Young children & 36 & 67 & 72 & 20 & 8 & $\begin{array}{c}139 \\
(301 ; 0-1,356)\end{array}$ \\
\hline School-aged children & 15 & 53 & 47 & 20 & 33 & $\begin{array}{c}552 \\
(994 ; 0-3,480)\end{array}$ \\
\hline Fishermen & 9 & 67 & 70 & 20 & 10 & $\begin{array}{c}64 \\
(95 ; 0-2,76)\end{array}$ \\
\hline \multirow{2}{*}{ Group } & \multicolumn{6}{|c|}{ 6-month } \\
\hline & $\mathrm{N}$ & Infection $(\%)$ & Light (\%) & Moderate (\%) & Heavy $(\%)$ & Mean EPG (SD, range) \\
\hline Mothers & 30 & 20 & 90 & 7 & 3 & $\begin{array}{c}44 \\
(151 ; 0-780)\end{array}$ \\
\hline Young children & 29 & 34 & 86 & 14 & 0 & $\begin{array}{c}29 \\
(59 ; 0-204)\end{array}$ \\
\hline School-aged children & $\mathrm{ND}$ & ND & ND & ND & ND & ND \\
\hline Fishermen & $\mathrm{ND}$ & ND & ND & ND & ND & ND \\
\hline
\end{tabular}

$\mathrm{ND}$, not determined

Young children were not asked about past infection and treatment, as children aged five years have not been routinely included in previously research and control operations in this community.

Despite the presence of the NCP, baseline infection levels were high (Table 2). In all groups, more than half of the individuals tested positive for the presence of S. mansoni eggs in the stools. While the majority of these were light infections, some groups, such as 33\% of school-aged children had heavy infections. Young children, who are not usually examined for infection, were found to be one of the highest prevalence groups with some children $(8.3 \%)$ harbouring heavy infections.

At the 6-month post-treatment follow-up, seven mother/child pairs were lost to follow-up. The demographics of the remaining mother-child cohort were not significantly different from those presented in Table 1 . Nearly a third of mothers and young children had become re-infected in the 6 -month period. The large majority of these were light intensity infections. Correspondingly, average EPG levels were one fifth of their baseline values.

At baseline, young children's infections were highly correlated with their mothers. Finding both mother and her young child infected accounted for $47 \%$ of the 36 pairs, while both were uninfected in $19 \%$ of the pairs; $14 \%$ of the pairs showed only the mother infected and in $19 \%$ only the young child was infected. The opposite situation was found at the 6-month follow-up as the majority of the mother-child pairs were not infected at this point. Of the 29 mother/child pairs that completed the infection examination at six months, only $3 \%$ of the pairs showed both mother and child infected, while both were uninfected in $51 \%$ of the cases; in $14 \%$ of the pairs only the mother was infected and in $31 \%$ only the young child was infected.

\section{GPS-derived exposure}

User acceptance was very high with only $8 \%$ (all mothers) reporting that the GPS device was felt to be uncomfortable. No participants reported that the device prevented them from performing regular activities inclusive of swimming. All GPS devices were returned at the end of three days and $98 \%$ said that they would be willing to participate in the experiment again. Out of the 97 participants, GPS data were successfully obtained from 85 participants, the difference explained by unit malfunctions.

Visualization of the GPS activity data revealed significant clustering around the village with considerable time spent along the shore, where infection can occur (Fig. 3). The figure shows each individual in a different colour illustrating that some individuals were more mobile than others. "Tentacles" of activity spread along the coast was observed for some partici- 

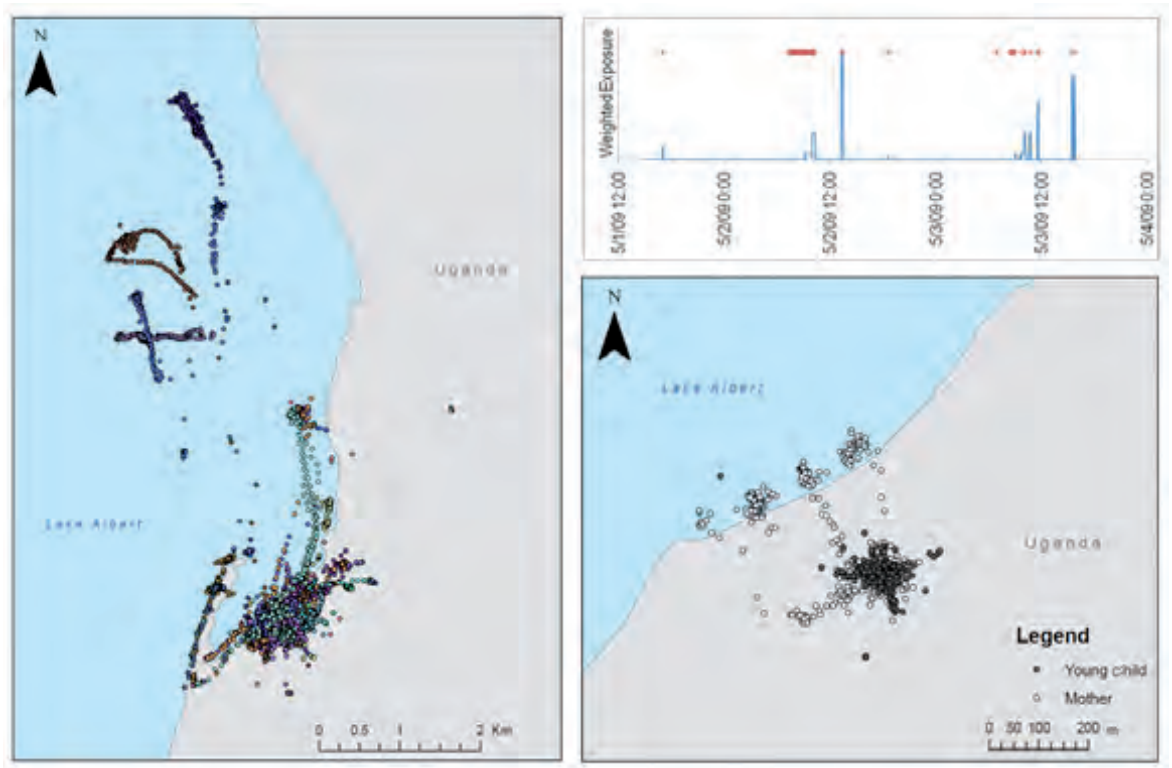

Fig. 3. GPS data from entire cohort plotted (left), enlarged region with GPS data for a mother-young child pair (bottom right) and time-series of weighted exposure for this mother (top right). The red crosses denote times when mothers entered the buffer zone and the column chart indicates the associated risk for these water contacts.

pants, while for fishermen in particular, these stretched out into the lake. Clear differences in activity and potential exposure patterns were found when the data for mother/child pairs were visualised in this way. As an example, one such pair (Fig. 3, right bottom) documented that the mother spent considerable time along the coast and thus was probably exposed to infection, while her young child's activity pattern remained concentrated away from coast.

Given the large number of GPS data points, visualization of activity patterns were aided by kernel density contours (Fig. 4). Note that there were fewer con- tours for the fishermen and school-aged children because of the smaller number of participants compared to the larger mother/child cohort. The contours for young children were highly concentrated at the village. Although the mothers' patterns were also concentrated at the village, comparing the largest contours for the young children with that of their mothers' suggests that the mothers' activities were slightly more dispersed and may have involved more time along the coast. The largest contour for school-aged children was similar to that of the mothers. Consistent with their occupation, fishermen had the most dis-
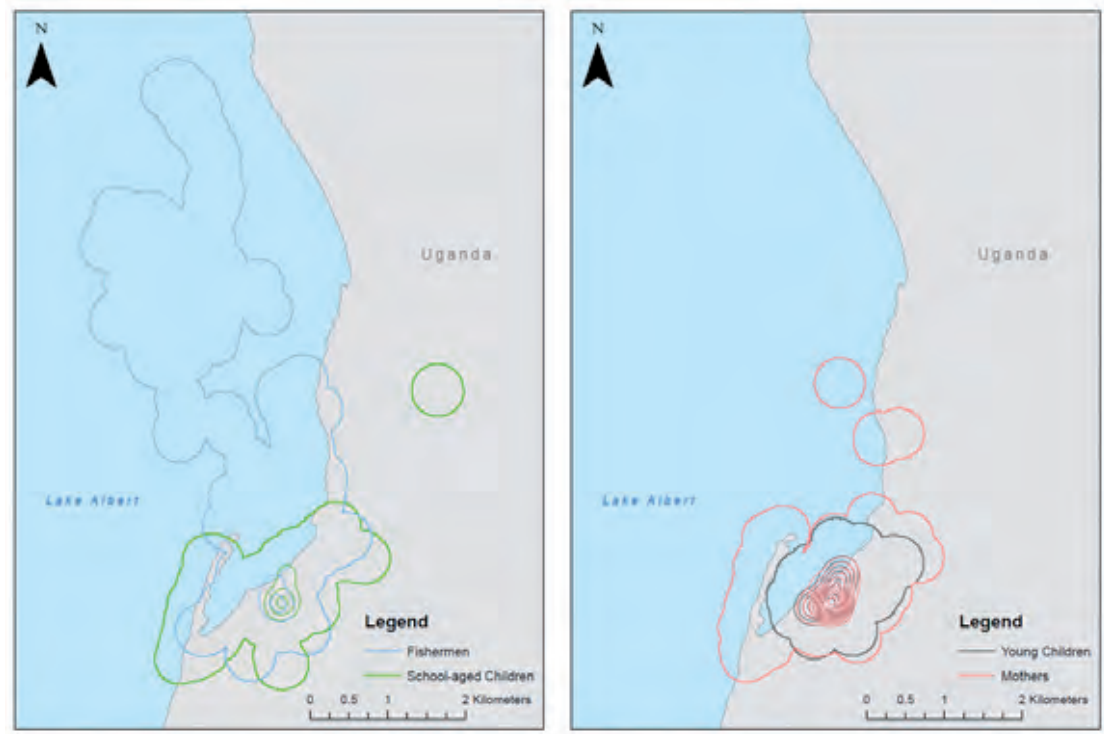

Fig. 4. Geographical distribution of activities within each GPS survey show some clear spatial similarities. 
Table 3. GPS-derived exposure minutes and weighted exposure score.

\begin{tabular}{lccc}
\hline Group & $\mathrm{N}$ & Exposure minutes mean $(\mathrm{SD} ;$ range $)$ & Weighted exposure score mean (SD; range) \\
\hline Mothers & 30 & $137(179 ; 0-743)$ & $3.7(2.1 ; 0-6.6)$ \\
Young children & 35 & $112(262 ; 0-1041)$ & $2.7(2.2 ; 0-6.9)$ \\
School-aged children & 11 & $124(160 ; 0-464)$ & $3.9(1.8 ; 0-6.1)$ \\
Fishermen & 9 & $300(440 ; 27-1430)$ & $5.1(1.1 ; 3.3-7.3)$ \\
\hline
\end{tabular}

persed activity patterns involving considerable time spent in the lake.

Based on coastline activity, exposures minutes and weighted exposure scores were computed for each of the four groups (Table 3). Fishermen had the highest mean exposure, followed by mothers, school-aged children and young children. The distribution of exposures was over-dispersed with larger variation in relation to the mean for all groups, particularly for young children. Differences in unweighted exposure between mothers and children were found to be significant $(\mathrm{P}=$ 0.039). Weighting the exposure based on cercarial shedding hours produced a different ranking of mean exposure, with school-aged children's exposures being slightly higher than mothers due to a higher proportion of exposure of school-aged children during periods of cercarial shedding than non-shedding periods. Weighted exposure differences between mother and child, however, were not found to be statistically significant $(\mathrm{P}=0.12)$.

The weighting scheme provides temporal context to an individual's water contact patterns. For example, one mother's activity patterns in Fig. 3 (bottom right) was plotted as a time series (Fig. 3, top right). The red marks at the top illustrate time spent along the shoreline, while the blue bars represent the weighted risk for those times. For this mother, some periods of exposure occurred too early or too late in the day when there were relatively few infective cercariae. Instead, relatively infrequent and short durations of exposure during mid-day resulted in elevated risk.

\section{GPS-derived exposure versus self-reported water con- tact}

GPS-derived weighted exposure was only moderately related to self-reported admission (yes/no) of lake water contact during the 3-day period the participant wore the device (Fig. 5). All fishermen and schoolaged children admitted to having water contact, though there was more variation in the degree of exposure among the latter group. Among the mothers, $82 \%$ admitted to having lake water contact. The median GPS-derived exposure for mothers reporting water contact was higher than those that did not $(3.5$ versus 0.99). Self-reported duration of water contact was positively correlated with GPS-derived exposure $(\mathrm{P}=0.01)$.

\section{Relationship between GPS-derived exposure and S. mansoni infection}

Infection at baseline was not significantly associated with GPS-derived exposure. Moreover, previous infection or treatment was not associated with baseline infection. However, there was an association between GPS-derived weighted exposure and infection at 6month follow-up for mothers and children [odds ratio $(\mathrm{OR})=2.1, \mathrm{P}=0.01,95 \%$ confidence interval $(\mathrm{CI}) 1.2$ - 3.7] (Table 4). A stronger relationship was found for young children alone $(\mathrm{OR}=4.4, \mathrm{P}=0.01,95 \% \mathrm{CI} 1.4$ $-14.0)$, though the relationship for mothers alone was not statistically significant. Infection at the 6-month follow-up was not significantly associated with age or sex but was strongly associated with baseline infection status $(\mathrm{OR}=13.0, \mathrm{P}=0.02,95 \%$ CI $1.6-108.0$, not shown in table).

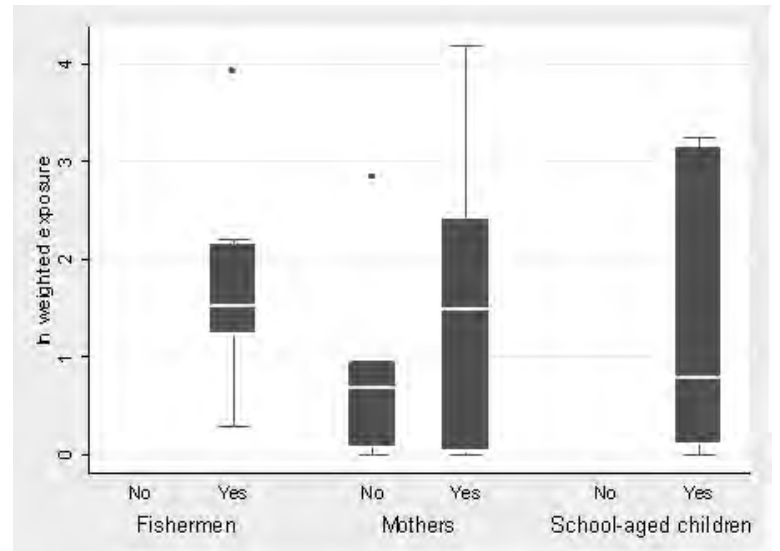

Fig. 5. Self-reported lake water contact (no/yes) versus GPSderived weighted exposure. Upon return of the device, each individual was asked how many times and for how long he/she had been to the lake with the device; this figure shows the variability between the correlation of this information and that which was derived from the GPS data. 
Table 4. Univariate infection relationships at baseline and at 6-month follow-up.

\begin{tabular}{|c|c|c|}
\hline Group & Baseline OR (P, 95\% CI) & 6-month OR (P, 95\% CI) \\
\hline Mothers and young children & $\mathrm{N}=65$ & $\mathrm{~N}=52$ \\
\hline ln (weighted exposure) & $1.00(0.91,0.66-1.6)$ & $2.10(0.01,1.2-3.7)$ \\
\hline Age & $0.99(0.44,0.96-1.0)$ & $0.98(0.30,0.94-1.0)$ \\
\hline Sex $(1=$ male $)$ & $0.65(0.41,0.23-1.8)$ & $0.86(0.82,0.23-3.2)$ \\
\hline Young children & $\mathrm{N}=36$ & $\mathrm{~N}=28$ \\
\hline ln (weighted exposure) & $1.40(0.32,0.70-2.9)$ & $4.40(0.01,1.4-14)$ \\
\hline Age & $1.30(0.48,0.65-2.5)$ & $1.00(0.94,0.49-2.2)$ \\
\hline Sex $(1=$ male $)$ & $0.28(0.11,0.06-1.3)$ & $0.25(0.1,0.048-1.3)$ \\
\hline Mothers & $\mathrm{N}=30$ & $\mathrm{~N}=24$ \\
\hline ln (weighted exposure) & $0.74(0.37,0.39-1.4)$ & $1.40(0.40,0.64-3.0)$ \\
\hline Age & $0.95(0.26,0.87-1.0)$ & $1.00(0.74,0.91-1.1)$ \\
\hline School-aged children & $\mathrm{N}=11$ & \\
\hline ln (weighted exposure) & $1.10(0.91,0.42-2.6)$ & ND \\
\hline Age & $0.86(0.51,0.56-1.3)$ & ND \\
\hline Sex $(1=$ male $)$ & $1.30(0.78,0.17-10)$ & ND \\
\hline Fishermen & $\mathrm{N}=8$ & \\
\hline ln (weighted exposure) & $3.60(0.30,0.32-41)$ & ND \\
\hline Age & $1.00(0.98,0.88-1.1)$ & ND \\
\hline
\end{tabular}

$\mathrm{ND}$, not determinated

\section{Social interaction and exposure}

A graph of the interactions between mothers-mothers, mothers-young children and young childrenyoung children is displayed in Fig. 6, with the status of infection at the 6-month follow-up indicated in purple. The four clusters in the network correspond to the four separate time periods of the GPS surveys. The number of unique interactions with other persons in the study and the total duration of interaction time, was computed for each study subject within the exposure zone. Both social interaction metrics were found to be correlated with exposure score (Pearson correlation 0.46 and 0.58 , respectively). On average, young children had the longest interaction times [mean 704, standard deviation (SD) 1,700 min] followed in order by mothers (mean 394, SD $722 \mathrm{~min}$ ), fishermen (mean 283, SD $359 \mathrm{~min}$ ), and school-aged children (mean 167 , SD $187 \mathrm{~min}$ ). Mothers tended to interact with the most people (on average four other persons in the exposure zone), followed by young children, fishermen and school-aged children in that order. Understandably, mothers tended to interact more with their own children $(\mathrm{M}=165.7, \mathrm{SD}=405.1 \mathrm{~min})$ than with other children $(\mathrm{M}=123.7, \mathrm{SD}=340.3 \mathrm{~min})$. Young children, however, interacted more with other young children $(\mathrm{M}=356.4, \mathrm{SD}=829.5 \mathrm{~min})$ than even their own mothers suggestive of gathering of the children, perhaps for safety while near the water.

\section{Discussion}

Despite the presence of a NCP, our study found considerable levels of infection and re-infection are occurring on this lake, especially within subpopulations that continue to come into contact with lake water for their livelihood and daily lives. This lakeside way of living has been documented for nearly a century (Worthington, 1929), and will not likely change with a NCP focused on morbidity reduction through drug treatment, rather than improved sanitation, changes in water contact and waste management behaviour. However, even with the reinfection levels found in our study, there is encouraging evidence that short term infection intensities decrease as a result of drug treatment. Young children ( $\leq 5$ years), often ignored in schistosomiasis control work, can obviously harbour heavy infections and may be particularly important to treat to reducing very early cumulative morbidity as they progress through childhood (Stothard and Gabrielli 2007a).

Interestingly, follow-up data reveal that infection rate for the mother group is lower than that of the 


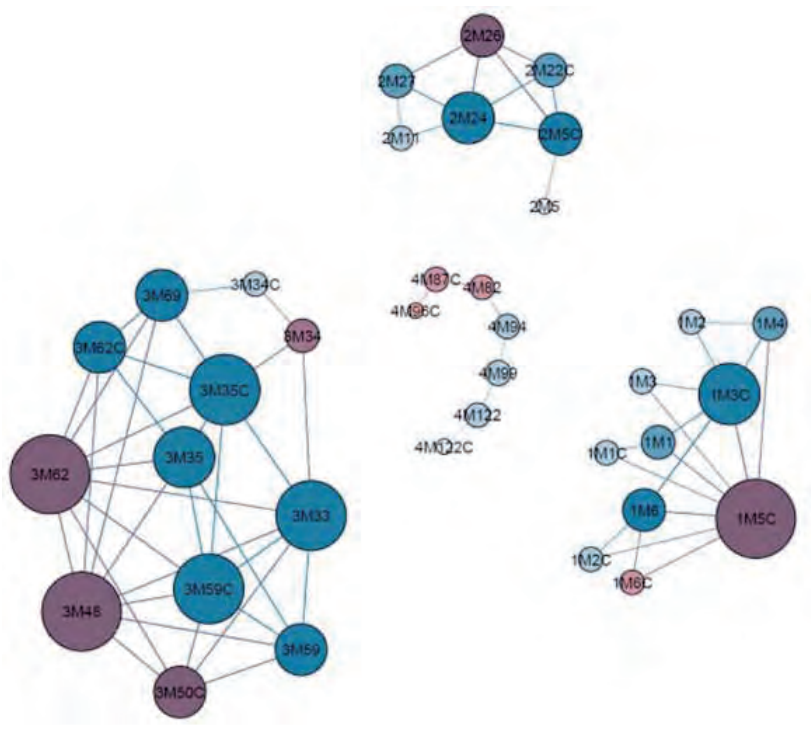

Fig. 6. GPS-derived social network of interpersonal interactions between mother-mother, mother-young child and young childyoung child, with the node size indicating number of interpersonal interactions (degree). Purple colour indicates infected individuals at 6-month follow-up, blue colour indicates the individual was infected at baseline (shades of colour indicate infection intensity). The node IDs indicate 4 discrete time periods (1-4) when GPS experiment was undertaken, the mother's ID (e.g. M62), and end in " $\mathrm{C}$ " if the node is a young child (e.g. 3M59C is the preschool-aged child of mother 3M59 which took part in the $3^{\text {rd }}$ GPS study). The presence of an edge between nodes indicates interaction (interaction time $>0$ ) between two individuals, therefore the more edges from a single individual indicate more interations.

young child group despite the mothers having higher water contact frequency and duration. This may be the result of the differential timings of water contact, whereby mothers go to the water more frequently and for longer periods of time, but these exposures may be taking place when snails are not shedding cercariae, as seen in Fig. 3 (right top) and as demonstrated in previous work by the authors (Stothard et al., 2011b). On the other hand, children are exposed (voluntarily or involuntarily through bathing behaviours) to lake water early in the morning when the infectious cercariae are abundant. Another explanation could be immunity; i.e. these results could be evidence of presence of an acquired immunity in the mothers, whereby increased exposure results in lower-than-expected infection rates; a phenomenon previously shown elsewhere (Black et al., 2010).

Whilst the mechanism of $S$. mansoni infection is well-understood as predominately resulting from cercarial contact with human skin, the ability to quantify this situation objectively has been an ongoing challenge within schistosomiasis research. The typical overdispersion of parasites as suggested by the infection intensity data for our study community may be explained by numerous factors that are related to exposure, but there are also confounding factors. These include the amount of water contact, whether it occurs in locations with infected snails (and at the specific time when cercariae are shed), how much skin contact with water, the presence of age-related factors (e.g. acquired immunity or changes in skin texture), and whether cercarial-inhibiting factors such as soap are present. While we were not able to consider all of these issues here, we have presented a novel use of wearable GPS dataloggers for objectively quantifying caercarial exposure.

GPS-based exposure assessment helps solve problems associated with self-reporting methods, including those related to recall or deliberate misreporting. Assessing variations in water contact from a selfreport survey instrument often requires a series of questions which, on one hand, can refine estimates of and provide context for exposure, but on the other hand requires more time and can be burdensome for the study participant. We found moderate correlation between self-reported admission of water contact and the GPS-derived exposure metric. Most notably, among those individuals who reported that they had water contact, there was considerable variability in the magnitude of exposure. This variability, which may be due to differing frequencies and durations of contact activity between individuals, as well as related to some individuals having contacts mainly during cercarial non-shedding periods versus some who have more mid-day contacts (as seen in Fig. 3). However, the responses to an additional survey question related to duration of water contact were found to be positively correlated with GPS-based exposures, suggesting that our exposure estimate has merit.

While a small number of data from our GPS units were lost due to malfunctioning, the units generally performed satisfactorily and were well-accepted by the participants. Given the low cost of these GPS devices (60 US\$) replacing lost or broken units in epidemiologic studies is generally not a problem. We note that in our current studies we relied exclusively on the GPS devices to estimate exposure, as opposed to earlier work in which GPS datalogging was paired with a follow-up survey to determine the nature of water contact activities at locations along the GPS trail (Seto et al., 2007b). As such, the approach was considerably less-burdensome upon study participants who only needed to return the GPS units. We found that user acceptance was high using this procedure, and nearly all reported that they would be willing to be monito- 
red again with the GPS dataloggers. Moreover, because all individuals felt that the device did not prevent them from conducting their regular activities, we believe that compliance with wearing the GPS should not disappear with time.

The use of GPS has many potential limitations in describing infection risk since it cannot address issues of acquired immunity, variations in body surface area contacted for different types of water-related activities or spatial variations in cercarial concentrations. Moreover, in our study we were not able to assess how exposure to domestic water (i.e. away from the immediate lakeshore) contributes to passive exposures (e.g. by bringing water from the lake into households to bathe children). Yet, even as a crude measurement of exposure, we found the GPS-based exposure metrics to be associated with short-term incidence of infection. The stronger relationship between exposure and infection in young children may reflect less immunity in younger populations. The lack of an association with baseline infection patterns may reflect that over time, practically everyone in the community has some exposure that can result in infection. Indeed, prevalence was quite high at baseline ( $>50 \%$ for all groups).

Another concern of the GPS approach is whether a relatively short (3-day) measurement of an individual's activity pattern is sufficient to characterise seasonal water contact patterns. Answering this definitively would require long-term monitoring, which is logistically infeasible. However, at the 6-month revisit to the study site, a subset of 11 individuals repeated the GPS experiment. Estimated unweighted and weighted exposures were found to be correlated with those at baseline (Pearson's correlation coefficients of 0.25 and 0.36 , respectively), suggesting that individuals may indeed be creatures of habit, with somewhat consistent water contact patterns over the course of a transmission season. Similar evidence of short-term exposure being related to longer term infection patterns has been found in other settings (e.g. in People's Republic of China), where assessed exposure was found to be related both to short-term $S$. japonicum infection as well as levels of endemic transmission at the individual and village-level (Seto et al., 2007b).

Using the data from the GPS, we were able to construct a social network of potential interpersonal interactions. We note that the extent of network is limited by the number of participants in the study, resulting in an underestimate of interactions. Nevertheless, based on a network of those individuals that did wear the GPS, we found a rather striking relationship between social interactions and schistosomiasis exposure.
While it makes inherent sense that social interactions would be related to communicable diseases, why such interactions would relate to an environmentally mediated parasitic disease raises some interesting hypotheses. First, common exposure events such as fetching water for domestic use may be done at the same time of the day by different families. Occupational work at the lake, including loading/unloading boats and drying fish may be done in teams, which involves groups of people interacting and being exposed together. It may also be that if an individual visits the lakeshore and meets other people, he/she may stay longer to socialise, hence increasing exposure. Young children may be cared for together and hence share exposure. From the GPS data we were able to observe some structure to the social interactions (e.g. mothers interact more with their own children than other children, while young children interact more with other young children). However, this is just a glimpse at what may be complex social networks that influence transmission. Additional detailed ethnographic studies of the social and cultural factors that affect both interpersonal interactions and exposure to schistosomiasis are warranted.

While we do not currently know the directionality of this association-whether interactions drives increasing exposure, or exposures inherently involve more social interactions-it is nevertheless worthwhile to speculate how knowledge of this association may be used for disease control. For instance, it may be possible in the future to leverage social interactions in health education and treatment programmes to maximize knowledge of risky behaviour and improve treatment coverage and compliance. Moreover, sustainable disease control programmes that aim to provide engineered environments (e.g. clean water, waste treatment, etc.) will need to carefully consider the ramifications of new technologies and find out whether they can provide for quality social interactions necessary to be culturally acceptable.

\section{Conclusion}

This study has clarified that, despite regular chemotherapy, there are the high levels of schistosomiasis infection in the community studied, a situation very probably mimicked around Lake Albert. It was demonstrated that infections among preschool-aged children are common and quickly return after 6 months of treatment confirming high levels of exposure through water contact. More broadly, we have demonstrated the use of wearable GPS dataloggers to 
assess both water contact exposure, which are directly related to parasite transmission, as well as to interpersonal interactions that may indirectly contribute to transmission.

\section{Acknowledgements}

We thank B. K. Wong for her assistance with social network graphing and editing of the paper. We wish to thank the children and mothers who took part in the study. We also thank the Ministry of Health of Uganda, Vector Control Division, and all their staff for their support during the "Schistosomiasis in Mothers and Infants" (SIMI) project. We would particularly like to acknowledge the work of the local Vector Control Division officers, Mr. Juma Nabonge, Mr. Ashuman Babyesiza, Mr. Perez Isingoma and Mr. Chris Byalero for making cohort studies possible in areas where populations are constantly migrating. $\mathrm{Mr}$. Chris Byalero, co-author of this paper, has regrettably passed away shortly before submission of the manuscript, and we would like to express our regret for the irreplaceable loss of a friend and colleague. JCSF and JRS would like to acknowledge Prof. David Rollinson and the Natural History Museum, London, for continued support during the SIMI project. We acknowledge financial support from the Wellcome Trust, UK (JRS).

\section{References}

Balen J, Stothard JR, Kabatereine NB, Tukahebwa EM, Kazibwe F, Whawell S, Webster JP, Utzinger J, Fenwick A, 2006. Morbidity due to Schistosoma mansoni: an epidemiological assessment of distended abdomen syndrome in Ugandan school children with observations before and 1-year after anthelminthic chemotherapy. Trans R Soc Trop Med Hyg 100, 1039-1048.

Barber TX, Silver MJ, 1968. Fact, fiction, and the experimenter bias effect. Psychol Bull 70, 1-29.

Bastian M, Heymann S, Jacomy M, 2009. Gephi: an open source software for exploring and manipulating networks. International AAAI Conference on Weblogs and Social Media. Black CL, Mwinzi PN, Muok EM, Abudho B, Fitzsimmons CM, Dunne DW, Karanja DM, Secor WE, Colley DG, 2010. Influence of exposure history on the immunology and development of resistance to human schistosomiasis mansoni. PLoS Negl Trop Dis 4, e637.

Brooker S, Kabatereine NB, Fleming F, Devlin N, 2008. Cost and cost-effectiveness of nationwide school-based helminth control in Uganda: intra-country variation and effects of scaling-up. Health Policy Plann 23, 24-35.

Brooker S, Kabatereine NB, Smith JL, Mupfasoni D, Mwanje MT, Ndayishimiye O, Lwambo NJS, Mbotha D, Karanja P, Mwandawiro C, Muchiri E, Clements ACA, Bundy DAP,
Snow RW, 2009. An updated atlas of human helminth infections: the example of East Africa. Int J Health Geogr 8, 42.

Chitsulo L, Engels D, Montresor A, Savioli L, 2000. The global status of schistosomiasis and its control. Acta Trop 77, 41-51.

Christakis NA, Fowler JH, 2008. The collective dynamics of smoking in a large social network. N Engl J Med 358, 2249 2258.

de Moira AP, Fulford AJC, Kabatereine NB, Kazibwe F, Ouma JH, Dunne DW, Booth M, 2007. Microgeographical and tribal variations in water contact and Schistosoma mansoni exposure within a Ugandan fishing community. Trop Med Int Health 12, 724-735.

Fleming FM, Fenwick A, Tukahebwa EM, Lubanga RGN, Namwangye H, Zaramba S, Kabatereine NB, 2009. Process evaluation of schistosomiasis control in Uganda, 2003 to 2006: perceptions, attitudes and constraints of a national programme. Parasitology 136, 1759-1769.

Gazzinelli A, Bethony J, Fraga LA, LoVerde PT, Correa-Oliveira R, Kloos H, 2001. Exposure to Schistosoma mansoni infection in a rural area of Brazil. I: water contact. Trop Med Int Health 6, 126-135.

Hotez P, Raff S, Fenwick A, Richards F, Molyneux DH, 2007. Recent progress in integrated neglected tropical disease control. Trends Parasitol 23, 511-514.

Johansen MV, Sacko M, Vennervald BJ, Kabatereine NB, 2007. Leave children untreated and sustain inequity! Trends Parasitol 23, 568-569.

Kabatereine NB, Ariho C, Christensen NO, 1992. Schistosoma mansoni in Pachwach, Nebbi district, Uganda, 40 years after Nelson. Trop Med Parasitol 43, 162-166.

Kabatereine NB, Brooker S, Koukounari A, Kazibwe F, Tukahebwa EM, Fleming FM, Zhang YB, Webster JP, Stothard JR, Fenwick A, 2007. Impact of a national helminth control programme on infection and morbidity in Ugandan schoolchildren. Bull World Health Organ 85, 91-99.

Kabatereine NB, Odongo-Aginya EI, Lakwo TL, 1996. Schistosoma mansoni along Lake Albert, Kibale district, western Uganda. East Afr Med J 73, 502-504.

Kabatereine NB, Vennervald BJ, Ouma JH, Kemijumbi J, Butterworth AE, Dunne DW, Fulford AJ, 1999. Adult resistance to schistosomiasis mansoni: age-dependence of reinfection remains constant in communities with diverse exposure patterns. Parasitology 118 , 101-105.

Katz N, Chaves A, Pellegrino J, 1972. A simple device for quantitative stool thick-smear technique in schistosomiasis mansoni. Rev Inst Med Trop São Paulo 14, 397-400.

Kazibwe F, Makanga B, Rubaire-Akiiki C, Ouma J, Kariuki C, Kabatereine NB, Booth M, Vennervald BJ, Sturrock RF, Stothard JR, 2006. Ecology of Biomphalaria (Gastropoda: Planorbidae) in Lake Albert, western Uganda: snail distributions, infection with schistosomes and temporal associations with environmental dynamics. Hydrobiologia 568, 433-444. 
Kazibwe F, Makanga B, Rubaire-Akiiki C, Ouma J, Kariuki C, Kabatereine NB, Vennervald BJ, Rollinson D, Stothard JR, 2010. Transmission studies of intestinal schistosomiasis in Lake Albert, Uganda and experimental compatibility of local Biomphalaria spp. Parasitol Int 59, 49-53.

Paz-Soldan VA, Stoddard ST, Vazquez-Prokopec G, Morrison AC, Elder JP, Kitron U, Kochel TJ, Scott TW, 2010. Assessing and maximizing the acceptability of global positioning system device use for studying the role of human movement in dengue virus transmission in Iquitos, Peru. Am J Trop Med Hyg 82, 723-730.

Rothenberg R, 2009. HIV transmission networks. Curr Opin HIV AIDS 4, 260-265.

Scott J, 1988. Social network analysis. Sociology 22, 109-127.

Scott JT, Diakhate M, Vereecken K, Fall A, Diop M, Ly A, De Clercq D, de Vlas SJ, Berkvens D, Kestens L, Gryseels B, 2003. Human water contacts patterns in Schistosoma mansoni epidemic foci in northern Senegal change according to age, sex and place of residence, but are not related to intensity of infection. Trop Med Int Health 8, 100-108.

Seto EY, Lee YJ, Liang S, Zhong B, 2007a. Individual and village-level study of water contact patterns and Schistosoma japonicum infection in mountainous rural China. Trop Med Int Health 12, 1199-1209.

Seto EYW, Knapp F, Zhong B, Yang C, 2007b. The use of a global positioning system-equipped vest for the assessment of water-contact patterns associated with schistosomiasis. Geospat Health 1, 233-241.

Sousa-Figueiredo JC, Pleasant J, Day M, Betson M, Rollinson D, Montresor A, Kazibwe F, Kabatereine NB, Stothard JR, 2010. Treatment of intestinal schistosomiasis in Ugandan preschool children: best diagnosis, treatment efficacy and sideeffects, and an extended praziquantel dosing pole. Int Healt 2, 103-113.

Spear RC, Seto E, Liang S, Birkner M, Hubbard A, Qiu D, Yang C, Zhong B, Xu F, Gu X, Davis GM, 2004. Factors influencing the transmission of Schistosoma japonicum in the mountains of Sichuan province of China. Am J Trop Med Hyg 70, 48-56.

Steinmann P, Keiser J, Bos R, 2006. Schistosomiasis and water resources development: systematic review, meta-analysis, and estimates of people at risk. Lancet Infect Dis 6, 411-425.
Stothard JR, Gabrielli AF, 2007a. Schistosomiasis in African infants and preschool children: to treat or not to treat? Trends Parasitol 23, 83-86.

Stothard JR, Gabrielli AF, 2007b. Response to Johansen et al.: Leave children untreated and sustain inequity! Trends Parasitol 23, 569-570.

Stothard JR, Sousa-Figueiredo JC, Betson M, Green HK, Seto EY, Garba A, Sacko M, Mutapi F, Vaz Nery S, Amin MA, Mutumba-Nakalembe M, Navaratnam A, Fenwick A, Kabatereine NB, Gabrielli AF, Montresor A, 2011a. Closing the praziquantel treatment gap: new steps in epidemiological monitoring and control of schistosomiasis in African infants and preschool-aged children. Parasitology 138, 1593-1606.

Stothard JR, Sousa-Figueiredo JC, Betson M, Seto EY, Kabatereine NB, 2011b. Investigating the spatial micro-epidemiology of diseases within a point-prevalence sample: a field applicable method for rapid mapping of households using low-cost GPS-dataloggers. Trans R Soc Trop Med Hyg 105, 500-506.

Utzinger J, Bergquist R, Xiao SH, Singer BH, Tanner M, 2003. Sustainable schistosomiasis control-the way forward. Lancet 362, 1932-1934.

Utzinger J, Raso G, Brooker S, de Savigny D, Tanner M, Ørnbjerg N, Singer BH, N'Goran EK, 2009. Schistosomiasis and neglected tropical diseases: towards integrated and sustainable control and a word of caution. Parasitology 136, 1859-1874.

van der Werf MJ, de Vlas SJ, Brooker S, Looman CW, Nagelkerke NJ, Habbema JD, Engels D, 2003. Quantification of clinical morbidity associated with schistosome infection in sub-Saharan Africa. Acta Trop 86, 125-139.

Vazquez-Prokopec GM, Stoddard ST, Paz-Soldan V, Morrison AC, Elder, JP, Kochel TJ, Scott TW, Kitron U, 2009. Usefulness of commercially available GPS data-loggers for tracking human movement and exposure to dengue virus. Int J Health Geogr 8, 68 .

WHO, 2002. Prevention and control of schistosomiasis and soil-transmitted helminthiasis: report of a WHO expert committee. WHO Tech Rep Ser 912, 1-57.

Worthington EB, 1929. The life of lake Albert and lake Kioga. Geogr J 74, 109-129. 